\title{
Prevention from swallowing or aspiration in dentistry for elderly patients
}

\begin{abstract}
Foreign body aspiration is a possible issue during dental treatment and may result in serious consequences, such as intestinal perforation. Its occurrence is relatively common when treating young children or the elderly. Many articles in dentistry have tackled the consequences and management whenever swallowing or aspiration occur, without focusing on prevention, which seems to be limited up until now. Thus, this paper aims at discussing methods for prevention of ingestion and aspirastion of foreign bodies during dental procedures.
\end{abstract}

Keywords: swallowing, aspiration, lip retractor, elderly patients
Volume 3 Issue 2 - 2018

\section{Gustavo Ismael,' Lanamar de Alameida, ${ }^{2}$ Tânia e Silva Pulicano Lacerda ${ }^{3}$ \\ 'Dentist, Universidade Paulista,Brazil \\ ${ }^{2}$ Radiologist at Hospital Paulistano, university of São Paulo, Brazil ${ }^{3}$ Associate Professor of Dentistry, Universidade Cidade de São Paulo, Brazil}

Correspondence: Tânia e Silva Pulicano Lacerda, Associate Professor of Dentistry, Universidade Cidade de São Paulo and São Leopoldo Mandic Graduate Center, Brazil,

Email tanialacerda@col.odo.br

\section{Introduction}

Aspiration or ingestion of instruments or materials used in dentistry is a relatively common risk during many dental procedures. Dentistry instruments are usually small and saliva makes them slippery. Thus, they may slip out from the operator's hand. ${ }^{1}$ Items that are more commonly accidentally inspired or swallowed include teeth, restorations and restorative materials, instruments, implant parts, rubber dam clamps, impression materials and crowns, ${ }^{2-5}$ It is important for the clinician to employ all the correct techniques to reduce the risk of this happening. The occurrence is relatively common when treating young children or the elderly. In older patients, the risk is higher due to the reduced gag reflex and other age-related general diseases, such as dementia or Parkinson's disease. ${ }^{6}$

The effects by such incident may be life-threatening, and involve damage to the digestive and breath tract, or can be a cause of obstruction, pneumonia, mediastinitis, peritonitis or sepsis. Usually instruments entered the gastrointestinal (GI) tract pass asymptomatically and atraumatically within 2 days to 4 weeks. However, there are many potential sites for impactions among them the ileocecal valve is the most common site.?

The aspiration of a foreign body may happen, and in these cases, there are no recognizable clinical signs of foreign body aspiration, nor of airway obstruction, not only diagnosis, but also treatment may become even more complicated. On physical examination, the most common findings include tachypnea, stridor, unilateral or bilateral decreased breath sounds, local wheezing and/or crackles, and sometimes fever. Unusual presentations consist of pneumomediastinum, subcutaneous emphysema and/or pneumothorax. ${ }^{6,8,9}$ In adults, the right main bronchus is the site most frequently involved..$^{9}$ This is explained by the anatomical features of the right main bronchus, which is wider in diameter, shorter in length and has a more direct extension of the trachea than the left bronchus. ${ }^{6}$

In a study ${ }^{10}$ among the French population it is reported that in all aspiration cases $(100 \%)$ required hospitalization compared to $36 \%$ for ingestion (166 cases of 464). This means that any ingested dental item, led to hospitalization in $36 \%$ of cases, but that all aspiration cases required hospitalization. ${ }^{11}$

\section{Prevalence and incidence}

Dental instruments are more frequently ingested than aspirated, retrospective and longitudinal studies of accidental ingestion and aspiration in large populations have reported that its incidence is around $0.004 \% .^{10,11}$ Another study showed similar numbers also with an incidence of $0.0037 \%$ per year. ${ }^{12}$ When comparing the prevalence between aspiration and deglutition, ${ }^{13} 8,7 \%$ of the case are aspirated and 91,3 of the case are related to deglutition. ${ }^{12}$ Obinata $\mathrm{K}$ et al. ${ }^{11}$ reported 23 cases of ingestion, and none of aspiration during dental procedures in the covered period.

Susini et al. ${ }^{10}$ has shown the numbers and types of foreign body accidents in dentistry and came up with, $27 \%$ were burs and $29 \%$ were prosthesis of the ingested items, endodontic files aspirated were $2.2 \%$ (one case in 44 ). Endodontic items represented 18\% (84 cases of 464 ) of all ingested items. Obinata $\mathrm{K}$ et al. ${ }^{11}$ analyzed retrospectively 23 cases of accidental ingestion during dental procedures, which occurred at the Center for Dental Clinics of Hokkaido University Hospital between 2006 and 2010. They came up with similar results that most foreign body ingestion accidents are related to dental metal crown and burs. Single-tooth cast or pre-fabricated restorations involving cementation had a higher risk of aspiration. ${ }^{3}$ This might be due to the fact that rubber dam is rarely used in prosthodontics compared to endodontics. ${ }^{14}$ 


\section{Risk factor}

The ingested objects were mostly metal restorations or prostheses. Ingestion occurred more frequently during treatment of lower molars and when procedures were being conducted by practitioners with less than 5 years of experience. Patient diseases associated with foreign body ingested items were reported by Obinata $\mathrm{K}$ et al. ${ }^{12}$ (2011). Out of 23 cases, 5 had cerebrovascular diseases (cerebral infarction in 3, Alzheimer's disease in 1, and Parkinson's disease in 1), 1 had undergone surgery for lower gingival carcinoma, and 1 had maxillary sinusitis. Patients who have undergone the removal of some type of malignant lesion in the oral cavity must be carefully assessed. Very often, these patients suffer from some kind of damage to their innervation, which may facilitate accidental ingestions. ${ }^{14}$ Previous surgeries in the GI tract increase the risk of perforation/obstruction. ${ }^{16}$ Patients who swallow foreign bodies most often are prisoners, psychotics, alcoholics, the senile and dental patients. ${ }^{1,17}$

The Mallampati score can be used to measure risk factor of the patient. It is based by asking the patient (in a sitting posture) to open his/her mouth and protrude the tongue as much as possible. ${ }^{18}$ The anatomy of the oral cavity is visualized; specifically, whether the base of the uvula, faucial pillar and soft palate are visible. Depending on whether the tongue is maximally protruded and/or the patient asked to phonate, the scoring may vary. Mallampati Scoring: Class I: Soft palate, uvula, fauces, pillars visible. Class II: Soft palate, uvula, fauces visible. Class III: Soft palate, base of uvula visible. Class IV: Only hard palate visible.

\section{Prevention from foreign body ingestion}

Prevention is of the highest importance when discussing accidental ingestion. Preventive steps include the placement of a gauze screen across the oropharynx and the use of a length of dental floss or suture material attached to small instruments in case they are dropped, adjusting chair position (sitting position prevents aspiration/ingestion and supine position increases the risk of swallowing) $)^{14,19}$ It is reported by Ratnaditya et al. ${ }^{18} 2014$ dental floss which is used to stabilize the hex drive is tied to a gold ring and the ring is put in position on the operator's finger ring to overcome sudden aspiration of fallen instrument (Figure 1). ${ }^{20}$

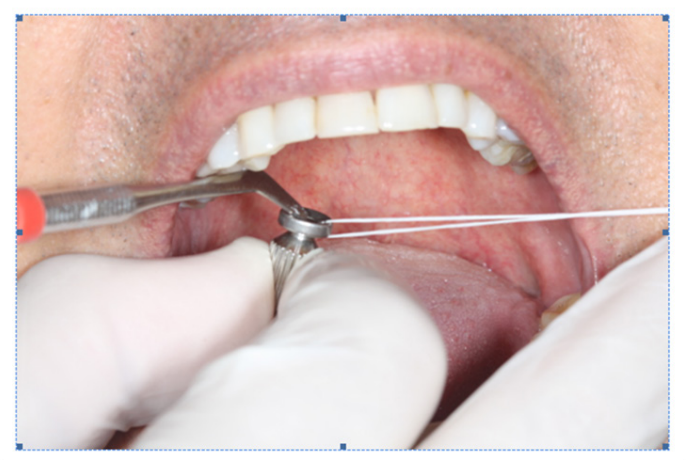

Figure I Dental floss preventing accidental ingestion during dental procedure.
Another possible step is the Lip retractor, which is associated with two neodymium's magnet (vertical draw Force $1.4 \mathrm{~kg}$, magnetic Grille $\mathrm{N} 42$, magnetic field in the distance of $0 \mathrm{~mm} 2230$ Gauss). This device can be used to prevent foreign bodies from being ingested, namely those which are attached by magnets. Its use is indicated for many dental procedures, maintaining mouth tissues retracted, allowing more visibility for the practitioner and preventing accidental ingestion. During the procedure, if there is any accidental fall of instruments, the lip retractor with the magnet will attract and hold the instrumental before entering the esophagus or trachea or even before touching the soft palate that triggers the gag reflex (Figure 2).

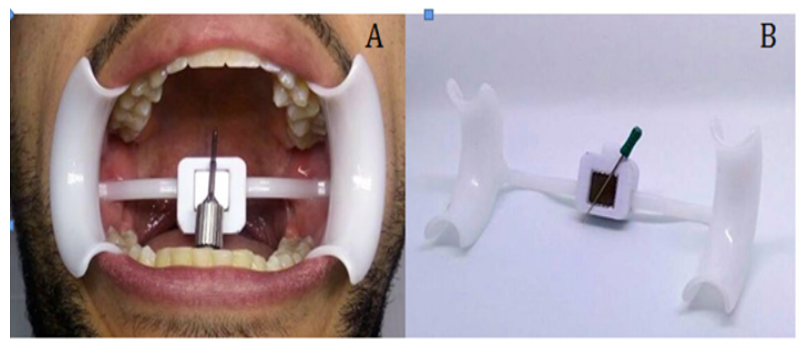

Figure 2 Dental Lip Retractor, with a magnet. A- It is shown an implant hex drive hold by the magnet on the lip retractor. B- Is an endodontic file hold by the magnet also).

\section{Discussion}

As it has been discussed here, during the dental procedures, ingestion of any instrument leads to serious complications. To prevent such accidental ingestions, standard practice guidelines need to be followed such as the use of gauze throat screens or floss ligatures. ${ }^{3}$ In many dental procedures (e.g. implant and protheses), usually application of rubber dam is not followed, which causes frequent ingestion of foreign bodies without the knowledge of the patient or the operator and even without any clinical signs. So, to prevent these conditions, it is always better to count the instruments before starting procedures and recounting them at the end of them. Accidental ingestion of dental instruments may sometimes cause serious complications. Most ingested foreign bodies pass through the GI tract successfully, taking anywhere from several days to several weeks. ${ }^{20,21}$ Radiographic evaluation and a fiber-rich diet is the initial protocol for the management of these cases. ${ }^{19}$ Radiographic examination is mandatory for differential diagnosis of the location, nature, and size of the foreign body. This can begin with the acquisition of CT scans or multiple films to include antero-posterior and lateral chest, lateral neck, and supine abdominal radiographs to complete the evaluation from the nasopharynx to the rectum. ${ }^{22}$ The object is usually passed. If serial radiographs depict the same location of foreign body or if there is a sign of abdominal tenderness or hemorrhage then most likely there is retention. Obstruction or intestinal perforation associated with abdominal pain and/or the presence of blood in the patient's feces are signs of intestinal perforation, instrument retention, or obstruction. In case of a patient presenting any of these signs, surgical removal is indicated (Figure 3) (Figure 4). If a foreign body was ingested/aspirated, the patient must be examined clinically and radiographically; diagnosis must be performed immediately by a specialist and the patient alongside his or her family members must be informed. 


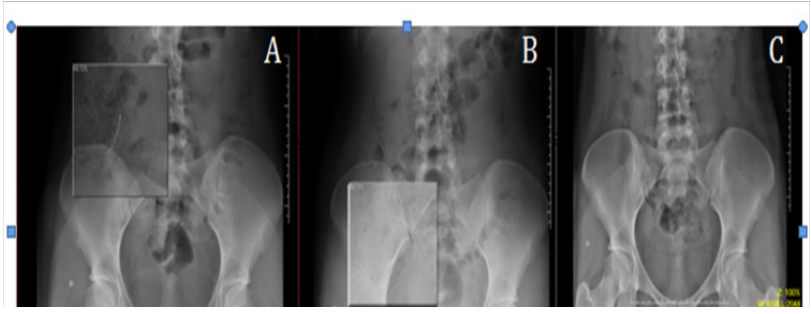

Figure 3 Abdominal radiographs obtained in dorsal decubitus demonstrating metallic material in the projection of a thin loop in the right iliac fossa (Case provided by Dr. Prof. Lanamar de Almeida). A, B and C are a follow up of the foreign body. A- Day I, B- Day 3, C- Day 5.

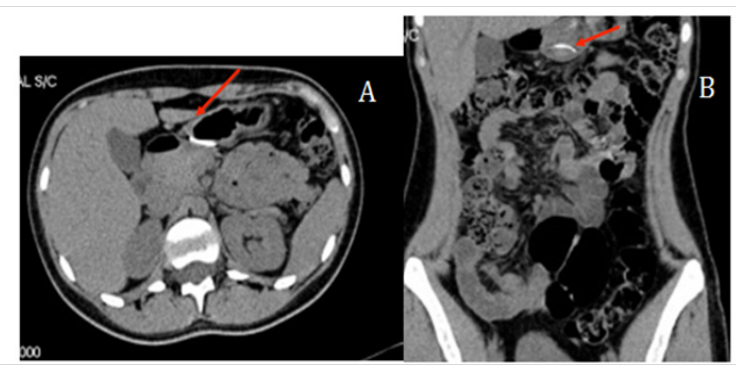

Figure 4 Axial and coronal images of computed tomography (CT) of the abdomen, showing metallic material inside the stomach, according to information collected, foreign body coming from dental braces. (Case provided by Dr. Prof. Lanamar de Almeida).

\section{Acknowledgements}

None.

\section{Conflict of interest}

The authors declare no conflict of interest.

\section{References}

1. Worthington P. Ingested foreign body associated with oral implant treatment: report of a case. Int J Oral Maxillofac Implants. 1996;11:679681.

2. Cameron SM, Whitlock WL, Tabor MS. Foreign body aspiration in dentistry: a review. J Am Dent As- soc. 1996;127(8):1224-1229.

3. Tiwana KK, Morton T, Tiwana PS. Aspiration and ingestion in dental practice: a 10-year institutional review. $J$ Am Dent Assoc. 2004;135(9):1287-1291.

4. Hodges ED, Durham TM, Stanley RT. Management of aspiration and swallowing incidents: a review of the literature and report of case. ASDC J Dent Child. 1992;59(6):413-419.

5. Pingarro ML, Mora SMJ, Anchez BRS, et al. Bronchial impaction of an implant screwdriver after accidental aspiration: report of a case and revision of the literature. J Oral Maxillo- fac Surg. 2010;14(1):43-47.
6. Fields RT, Schows SR. Aspiration and ingestion of foreign bodies in oral and maxillofacial surgery; a review of the literature and report of five cases. J Oral Maxillofac Surg. 1998;56(9):1091-1098.

7. Zitzmann NU, Elsasser S, Fried R, et al. Foreign body ingestion and aspiration. Oral Surg Oral Med Oral Pathol Oral Radiol Endod. 1999; 88(6):657-660.

8. Maleki M, Evans WE. Foreign-body perforation of the intestinal tract. Arch Surg. 1970;101(4):475-477.

9. Bergermann M, Donald PJ, Wengen DF. Screwdriver aspiration. A complication of dental implant place- ment. Int J Oral Maxillofac Surg. 1992;21(6):339-341.

10. Susini, L. Pommel, Camps. Accidental ingestion and aspiration of root canal instruments and other dental foreign bodies in a French population. International Endodontic Journal. 2007;40(8):585-589.

11. Obinata K, Satoh T, Mohammad AT, et al. An investigation of accidental ingestion during dental procedures. Journal of Oral Science. 2011;53(4):495-500.

12. Rashed MA. A method to prevent aspiration or ingestion of cast post and core restorations. Journal Pros- thetic Dentistry. 2004;91(5):501-502.

13. Souza JG, Schuldt FG, Pereira NAR, et al. Accident in Implant Dentistry: Involuntary Screwdriver Ingestion during Surgical Procedure. A Clinical Report. Journal of Prosthodontics. 2012; 21(3):191-193.

14. Henderson CT, Engel J, Schlesinger P, Foreign body ingestion: review and suggested guidelines for management. Endoscopy. 1987;19(2):68-71.

15. Reilly J, Tompson J, MacArthur C, et al. Pediatric aerodigestive foreign body injuries are complications related to timeliness of diagnosis. Laryngoscope. 1997;107(1):7-20.

16. Mallampati SR, Gatt SP, Gugino LD, et al. A clin- ical sign to predict difficult tracheal intuba- tion: a prospective study. Can Anaesth Soc $J$. 1985:32(4):429-434.

17. Barkmeier WW, Cooley RL, Abrams H. Prevention of swallowing or aspiration of for- eign objects. J Am Dent Assoc. 1978;97(3):473-476.

18. Ratnaditya A, Ravuri S, Tadi DP, et al. A simpli ed method of preventing implant hex drive from aspiration or accidental swallowing during stage two implant recovery. J Int Soc Prevent Communit Dent. 2014;4(1):S2325 .

19. Govila CP. Accidental swallowing of an endodontic instrument. A report of two cases. Oral Surg Oral Med Oral Pathol. 1979;48(3):269-271.

20. Lyons MFII, Tsuchida AM. Foreign bodies of the gastrointestinal tract. Med Clin North Am. 1993;77(5):1101-1114.

21. Hisanaga R, Hagita K, Nojima K, et al. Survey of accidental ingestion and aspiration at Tokyo Dental College Chiba Hospital. Bull Tokyo Dent Coll. 2010;51(2):95-101.

22. Sugawara C, Takahashi A, Maeda N, et al. An investigation of accidental aspiration and swallowing during the dental treatment at Tokushima University Dental Hospital. 2007;19:255-262.

23. Samarasam I, Chandran S, Shukla V, et al. A missing denture's misadventure!. Dis Esophagus 2006;19(1):53-55. 